\title{
Diagnostic Accuracy of Bronchoalveolar Lavage in Lung Lesions
}

\author{
Mridula. D. Shenoy*, Padma Shetty K and Jayaprakash Shetty K \\ Department of Pathology, K.S. Hegde Medical Academy, Karnataka (India)
}

\begin{abstract}
Background: Suspicious lesions in the lung on radiology need further evaluation. Bronchoalveolar lavage (BAL) is a simple diagnostic procedure which can be done on an outpatient basis. Cells obtained from bronchial and alveolar spaces can give a definite diagnosis in cases of malignancies and infections.

Methods: This is a prospective study done in Department of Pathology, K.S. Hegde Medical Academy between June 2014 and June 2015. 47 patients underwent BAL out of which 33 patients underwent simultaneous bronchoscopic biopsy. BAL fluid was centrifuged immediately and 4 smears were made from the sediment. Biopsy specimens were sent to the histopathology lab in $10 \%$ formalin.

Results: Of the 47 patients who underwent BAL, 18 patients had benign lesions and 22 cases were malignant. 7 cases were inadequate for diagnosis. Among the 47 patients only 33 patients had a conclusive histopathological diagnosis. Out of this 33 patients, 15 cases diagnosed as benign on BAL. However, on correlation with histopathology 7 were found to be malignant. Malignant cases on BAL were proved by histopathological examination (HPE). Overall efficacy for diagnosing malignant lesions in our study showed a sensitivity of $72 \%$, specificity of $100 \%$, positive predictive value of $100 \%$ and negative predictive value of $53.3 \%$

Conclusion: BAL shows good sensitivity, specificity and accuracy in diagnosing lung lesions. Benign cases needs to be confirmed by biopsy. Thus a combination of both BAL and HPE is always beneficial.
\end{abstract}

Keywords: Bronchoalveolar Lavage, Bronchoscopic Biopsy, Lung Carcinoma, Sensitivity

\section{Introduction}

Radiologically suspicious lung lesions must undergo further evaluation. ${ }^{[1]}$ Bronchoalveolar lavage is a low cost diagnostic procedure which can be done on an outpatient basis. It is a well tolerated and relatively safe investigation where saline wash of the bronchial tree is done to obtain cells for evaluation. It was first introduced in 1970 and became a diagnostic tool in india in the year 1994. ${ }^{[2]}$ Biopsy is a rapid, sensitive and inexpensive prodecure for diagnosing benign and malignant lesions. ${ }^{[3]}$ Bronchoscopic biopsies are taken with the help of a flexible biopsy needle and provide a confirmatory diagnosis. This study is conducted to evaluate the diagnostic accuracy between BAL and histopathology.

\section{Materials and Methods}

This is a prospective type of study conducted in the Department of Pathology, K.S. Hegde Medical Academy, Mangalore. This study was carried out over a period of one year, from June 2015 to June 2016. 47 patients underwent BAL, out of which bronchoscopic biopsy was performed in only 33 patients. The procedure was performed by the pulmonologists and then sent to the lab where it was processed. BAL samples were centrifuged immediately. The supernatant was discarded and 4 smears were made from the sediment. 2 smears were stained by PAP stain while the other 2 were stained with leishman stain. Biopsies were sent in containers containing $10 \%$ formalin to the histopathology lab where it was processed and stained. All the biopsy slides were stained by hematoxylin and eosin. Special stains were performed if needed. Statistical analysis was done with the help of SPSS version 21.

\section{Results}

47 patients underwent BAL fluid examination, however bronchoscopic biopsy was done in only 33 patients. Of the 47 cases, 18 cases were reported as benign, 22 malignant and 7 were inadequate for diagnosis. Among the 33 patients who underwent both BAL and HPE, 18 cases were found to be malignant on BAL which was confirmed by biopsy. The remaining 15 cases were reported as benign on BAL out of which 7 cases turned out to be malignant on HPE. The overall efficacy of BAL in diagnosing malignant lesions in our study showed a sensitivity of $72 \%$, specificity of $100 \%$, positive predictive value of $100 \%$ and negative predictive value of $53.3 \%$. Agreement between the 2 methods showed a kappa value of 0.555 . Overall the result was highly significant with $p$ value $<0.001$ 
Table 1: correlation of BAL with biopsy in comparison with other studies

\begin{tabular}{|c|c|c|c|}
\hline Authors & Sensitivity & Specificity & Diagnostic accuracy \\
\hline Bhat N et al $(2015)^{[4]}$ & $35.5 \%$ & $78.16 \%$ & $42.43 \%$ \\
\hline Tuladhar et al $(2011)^{[6]}$ & $66.7 \%$ & - & - \\
\hline Gaur DS et al $(2007)^{[5]}$ & $39.4 \%$ & $89.6 \%$ & $71.40 \%$ \\
\hline Wongsurkait et al $(1998)^{[8]}$ & $46.7 \%$ & - & - \\
\hline Pirozynski et al (1992) $)^{[7]}$ & $64.8 \%$ & - & - \\
\hline Present study & $72 \%$ & $100 \%$ & $78.79 \%$ \\
\hline
\end{tabular}

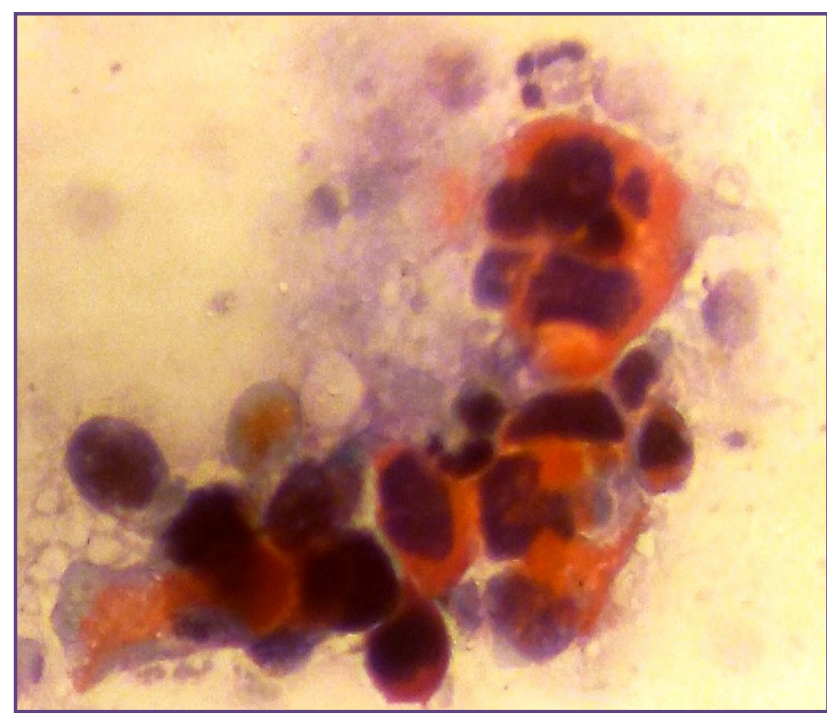

Fig 1: BAL showing malignant squamous cells (PAP stain, $\mathbf{x 1 0 0 )}$

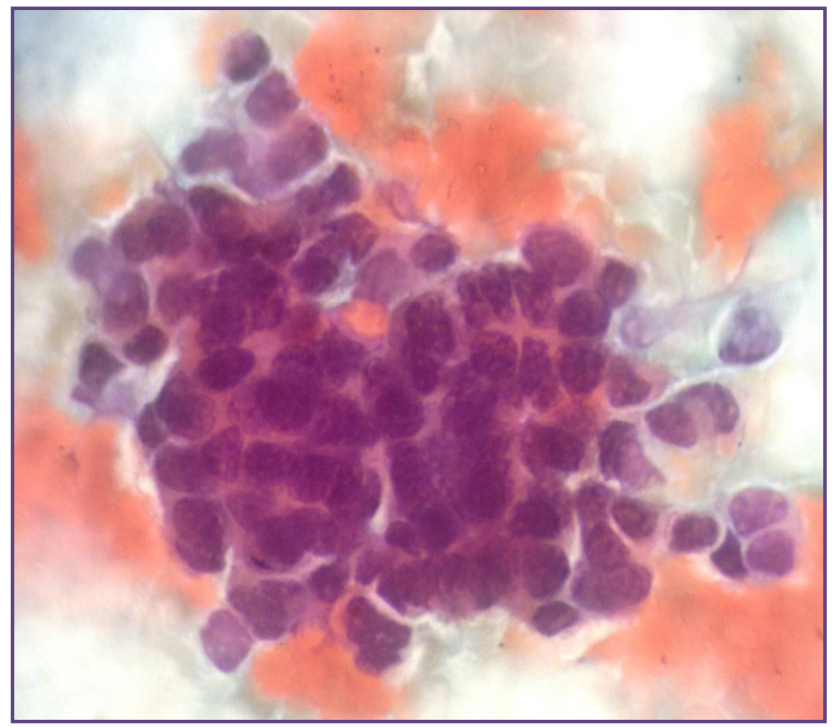

Fig 2: BAL showing small cell carcinoma with nuclear molding (PAP stain, $\mathrm{x} 40$ )

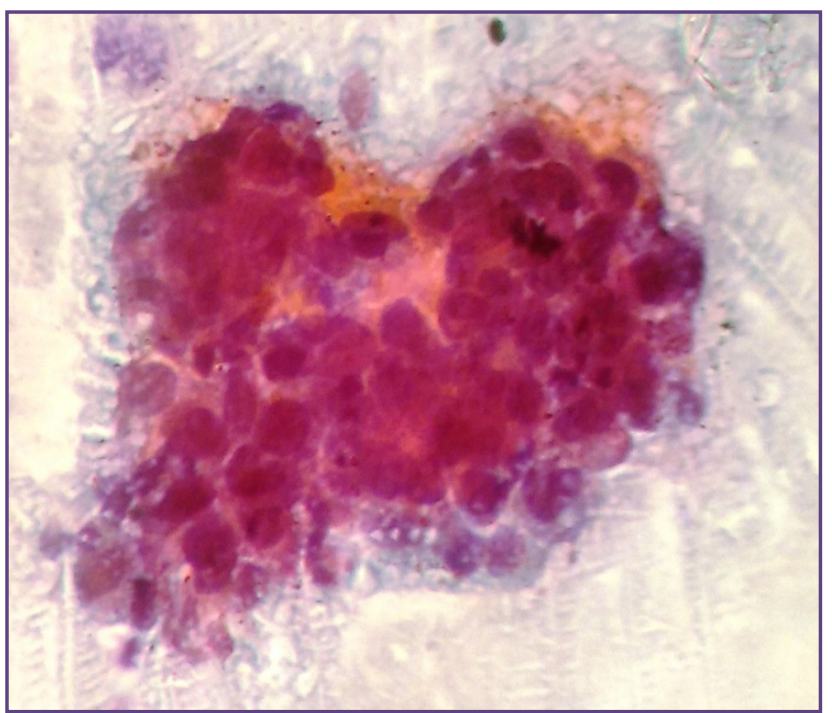

Fig 3: BAL showing cluster of malignant cells (adenocarcinoma) (PAP stain, $x 40$ )

\section{Discussion}

Even after conventional diagnostic evaluation by bronchoscopy and/or CT guided biopsy, patients with undiagnosed radiologically suspicious tumour in the lung pose a challenge to clinician. ${ }^{[3]} \mathrm{BAL}$ is a relatively safe and useful procedure for evaluation and has minimal complications. Even though BAL is considered slightly inferior to bronchial biopsy it can give a definitive diagnosis in infection and malignant cases. ${ }^{[4]}$ Our study deals with the diagnostic accuracy of BAL fluid examination in cases obtained over a period of one year. 47 samples were received in the year June 2015 to June 2016. The age ranged from 22 to 88 yrs with the mean age being 61.7 yrs. Among the 47 cases, 7 were inadequate for diagnosis. Of the remaining 40 cases, 18 were benign and 22 were malignant. Among the malignant cases, 4 were poorly differentiated carcinomas, 3 non small cell lung carcinomas (not otherwise specified), 3 adenocarcinomas and 2 squamous cell carcinomas. On histopathologic 
examination the 4 poorly differentiated carcinomas were further subclassified into 2 SCC, 1 Adenocarcinoma and the other was inadequate for diagnosis.

Of the 47 patients, 33 patients underwent simultaneous bronchoscopic biopsy. Out of the 15 benign cases diagnosed as BAL, 7 cases turned out to be false negatives. 6 cases which were reported as having no malignancy showed malignant cells on biopsy. 1 case which was diagnosed as inflammatory lesion on BAL turned out to be adenocarcinoma on histopathological examination. No false positive cases were observed in our study. We obtained a sensitivity of $72 \%$, specificity of $100 \%$ and diagnostic accuracy of $78.79 \%$.

This diagnostic accuracy was comparable with study done by Gaur DS et al (2007) ${ }^{[5]}$ who conducted a study on 196 cases obtained over a 5 year period. He obtained a sensitivity of $39.4 \%$, specificity of $89.6 \%$ and a diagnostic accuracy of $71.4 \%$. The lower sensitivity rate in this study was attributed to the fact that only a single sample of BAL as they felt that multiple samplings cause inconvenience to the patients. In contrast, Bhat $\mathrm{N}$ et al $(2016)^{[4]}$ conducted a study on 902 clinically suspected cases. Tumour was found by BAL method in 301 patients and by biopsy in 760 patients. They found a lower sensitivity and diagnostic accuracy of $35.5 \%$ and $42.43 \%$ respectively. The reason for lower accuracy may be due to the large number of false negative cases in their study which was 490 . Study done by Tuladhar et al (2011) ${ }^{[6]}$ on 50 patients showed BAL having better sensitivity than bronchial washings and brushings combined. Pirozynski et al (1992) $)^{[7]}$ did a study on 145 patients of which 94 cases by BAL showed malignant cells. Wongsurkait et al $(1998)^{[8]}$ study on 55 radiological suspicious patients showed malignancy in 30 patients. BAL showed malignant cells in 14 cases out of the $30 \mathrm{~m}$ positive cases. The studies done by Tuladhar et al (2011) ${ }^{[6]}$ Pirozynski et al (1992) $)^{[7]}$ and Wongsurkait et al $(1998)^{[8]}$ showed a sensitivity of $66.7 \%, 64.8 \%$ and $46.7 \%$ respectively to determine malignant cells on BAL.

\section{Conclusion}

Lung cancer is the most commonly diagnosed malignancy and the most common cause of mortality among cancer related deaths all over the world. Imaging modalities and cytologic examination help in evaluation and diagnosis of patients with intrathoracic lesions. BAL is an inexpensive procedure and can be done as an outpatient basis. BAL shows good sensitivity, specificity and accuracy in diagnosing lung lesions. Combinations of both cytologic and histopathologic examination have proved to be beneficial in diagnosing suspicious lesions.

\section{References}

1. Eckardt J, Petersen HO, Hakami-Kermani A, Olsen KE, Jorgensen OD, Licht PB. Endobronchial ultrasoundguided transbronchial needle aspiration of undiagnosed intrathoracic lesions. Interact Cardiovasc Thorac Surg. 2009 Aug 1;9(2):232-5.

2. Radha S, Afroz T, Prasad S, Ravindra N. Diagnostic utility of bronchoalveolar lavage. J Cytol. 2014 Jul;31(3):136

3. Naqvi H, Edhi M, Aslam M, Faridi N. Spectrum of intrathoracic lesion detected by computed tomography guided fine needle aspiration biopsy.International Archives of Medicine. 2013; 6(4):1-5

4. Bhat N, Nazeir M, Bashir H, Bashir N, Farooq S, Fatima K, et al. Correlation of bronchial biopsy with bronchoalveolar lavage in lung malignancies. Int J Res Med Sci. 2016;428-35.

5. Gaur DS, Thapliyal NC, Kishore S, Pathak VP. Efficacy of bronchoalveolar lavage and bronchial brush cytology in diagnosing lung cancers. J Cytol. 2007;24:73-7.

6. Tuladhar A, Panth R, Joshi AR. Comparative analyses of cytohistologic techniques in diagnoses of lung lesions. Journal of Pathology of Nepal.2011;1:126 -30

7. Pirozynski M. Bronchoalveolar lavage in the diagnosis of peripheral primary lung cancer. Chest 1992;102(2):372-4.

8. Wongsurakiat $\mathrm{P}$, Wongbunnate S, Dejsomritrutai W, Charoenratanakul S, Tscheikuna J, Youngchaiyud P, et al. Diagnostic value of bronchoalveolar lavage and post bronchoscopic sputum cytology in peripheral lung cancer. Respirology. 1998;3(2):131-7.

*Corresponding author:

Dr Mridula. D. Shenoy, 5-4-180/6(3), 'Prabhadham', kodialguthu west, mangalore (India)- 575003

Phone: +918105866651

Email: dr.mridulashenoy@gmail.com

Financial or other Competing Interests: None.

Date of Submission : 26.05.2017

Date of Acceptance : $\mathbf{3 0 . 1 0 . 2 0 1 7}$

Date of Publication : 28.01.2018 